\title{
AgNORs in the myocardium in ischaemic heart disease complicated by heart failure: a postmortem study
}

\author{
N N Mamaev, A Y Gudkova, K K Amineva
}

\begin{abstract}
Aim-To evaluate the interphase ribosomal RNA cistron activity of cardiomyocytes in cases with ischaemic heart disease complicated by heart failure.

Methods-Nucleoli were investigated in postmortem myocardium samples from 31 cases with ischaemic heart disease (mean (SEM), 57.4 (6.5) years) with or without severe heart failure (18 and 13, respectively) and from eight healthy people who died in accidents (mean (SEM) 25.3 (4.0) years). Myocardium obtained within one hour after death was frozen in liquid nitrogen. Silver staining for nucleolar organiser regions (AgNOR) was performed with a standard procedure and the mean score obtained. On the basis of these data, the average number of AgNORs per nucleus was determined. The Student's $t$ test was used to compare groups.

Results-Compared with controls, the mean numbers of AgNORs per nucleus in cardiomyocytes from ischaemic heart disease patients not complicated with severe heart failure were higher $(8.0 \quad v \quad 9.9$; $p<0.05$ ), but cases with severe heart failure had a progressive decrease in cardiomyocyte AgNORs. A difference in AgNOR numbers between cases with different ischaemic heart disease courses was found when cases with the same New York Heart Association (NYHA) functional stage III of heart failure were studied. Conclusions-The significant decrease of AgNORs in cardiomyocytes from cases with severe ischaemic heart disease complicated by heart failure seems to be connected with cardiomyocyte adaptation (a variant of hibernation) to a diminished circulation that, hypothetically, may affect the level of ribosomal RNA synthesis. (f Clin Pathol: Mol Pathol 1998;51:102-104)
\end{abstract}

Keywords: ischaemic heart disease; heart failure; nucleolar organiser regions; silver staining

Many chronic cardiac disorders, including ischaemic heart disease complicated by heart failure, are characterised by a reduced expression of mRNA and lowered concentrations of the contractile and cytoskeletal proteins. ${ }^{1-6}$ On the basis of these observations, it was suggested that these alterations are secondary to changes at the transcriptional level, or even higher. ${ }^{5}$ However, this has not been studied in heart disease. Accordingly, we investigated the ischaemic heart disease cardiomyocyte protein synthesising machinery by means of the inexpensive and fast nucleolar protein silver staining method (AgNOR), ${ }^{7-9}$ which assesses ribosomal cistron activity. A significant decrease in AgNOR scores was seen in cardiomyocytes from cases with a severe course of ischaemic heart disease, especially when complicated by heart failure. This suggests that cardiomyocytes react to ischaemia with upregulation of ribosomal cistron transcription.

\section{Methods}

In each case, a part of the left ventricular free wall was obtained from necropsied hearts within one hour of death. These comprised an ischaemic heart disease group $(n=31$ (eight male and 23 female); mean (SEM), 57.4 (6.5) years) and a control group ( $n=8)$, healthy people who had died in accidents (mean (SEM), 25.3 (4.0) years).

The duration of the disease ranged from one to 16 years (mean (SEM), 5.8 (2.3) years). Nineteen of 31 patients had associated moderate arterial hypertension. All patients had clinical signs of heart failure for a mean (SEM) of 2.2 (1.5) years, which was New York Heart Association (NYHA) class I/II (10 cases), class III (16 cases), and class IV (five cases).

On the basis of their clinical course, the patients were divided into two groups. The first consisted of 13 cases with stable angina pectoris. They were characterised by the presence of angina pectoris of functional classes II or III; four patients had a history of myocardial infarction.

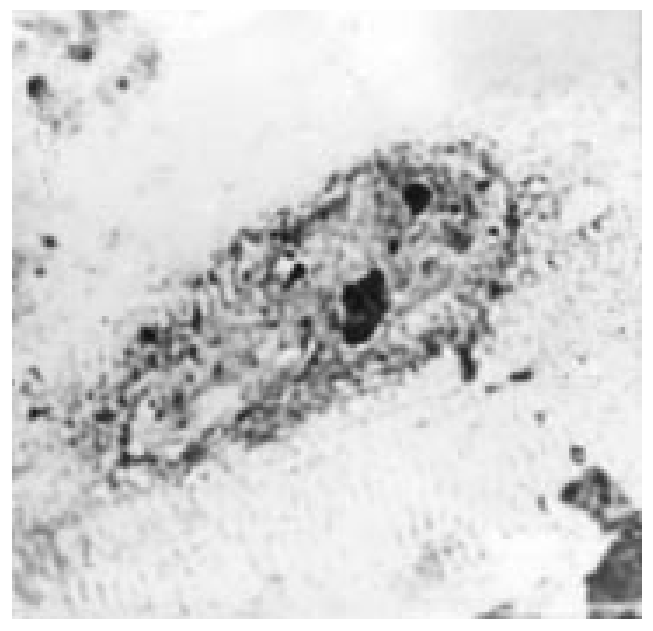

Figure 1 Silver stained cardiomyocyte in a necropsy sample from a case with ischaemic heart disease not complicated by severe heart failure (original magnification $\times 1000$ ). 

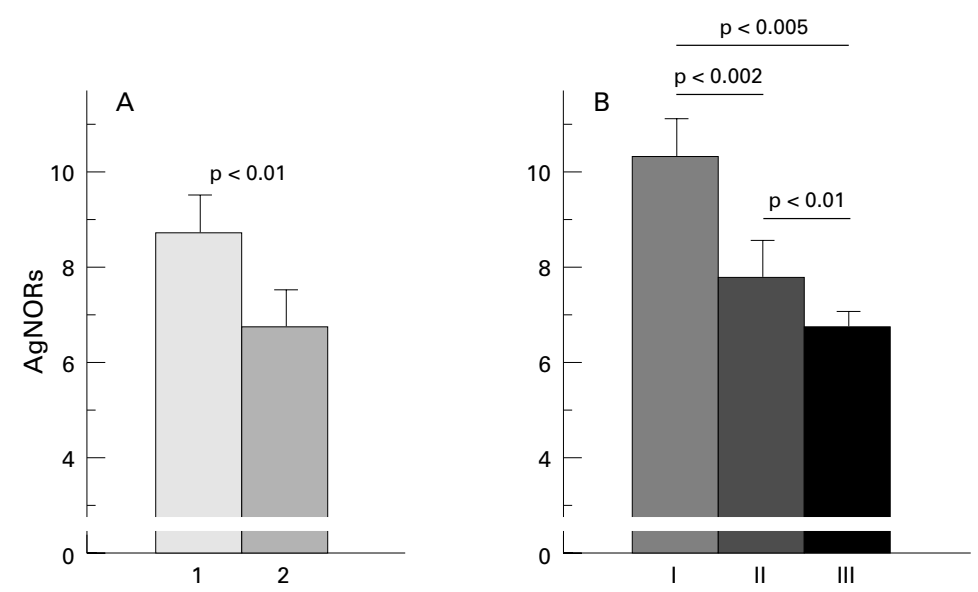

Figure 2 The mean numbers of AgNORs in cardiomyocyte nucleoli of postmortem myocardium from cases with a stable (A1) or progressive (A2) course of ischaemic heart disease but with a similar stage (III) of heart failure, and that with different NYHA functional stages of heart failure (B). I, II, III correspond to heart failure stages I/II, III, and $I V$, respectively.

The second study group comprised 16 patients with a more malignant and progressive course of the disease (unstable angina pectoris). Fifteen of these had sustained an acute myocardial infarction before hospital admission, while seven had sustained more than one documented myocardial infarction in the past.

The myocardial samples obtained at necropsy were frozen in liquid nitrogen. Cryostat sections $(10 \mu \mathrm{m}$ thick) were air dried, fixed in methanol:acetic glacial acid mixture (3:1) for 15 minutes, and rinsed thoroughly with distilled water. After repeated air drying, the sections were put into $2 \mathrm{~N}$ formic acid for 10 minutes, rinsed again, and impregnated with a mixture of a $50 \%$ aqueous solution of silver nitrate with gelatin at $68-70^{\circ} \mathrm{C}$, following the method of Howell and Black, ${ }^{10}$ with slight modification. ${ }^{7}$ The preparations were rinsed again thoroughly with distilled water and then counterstained with $2 \%$ buffered ( $\mathrm{pH}$ 6.8) Giemsa stain for 20 seconds, dehydrated, cleared, and mounted. At least 100 nuclei of silver stained cardiomyocytes were examined to count the numbers of AgNORs in nucleoli (fig 1). On the basis of these data, the average
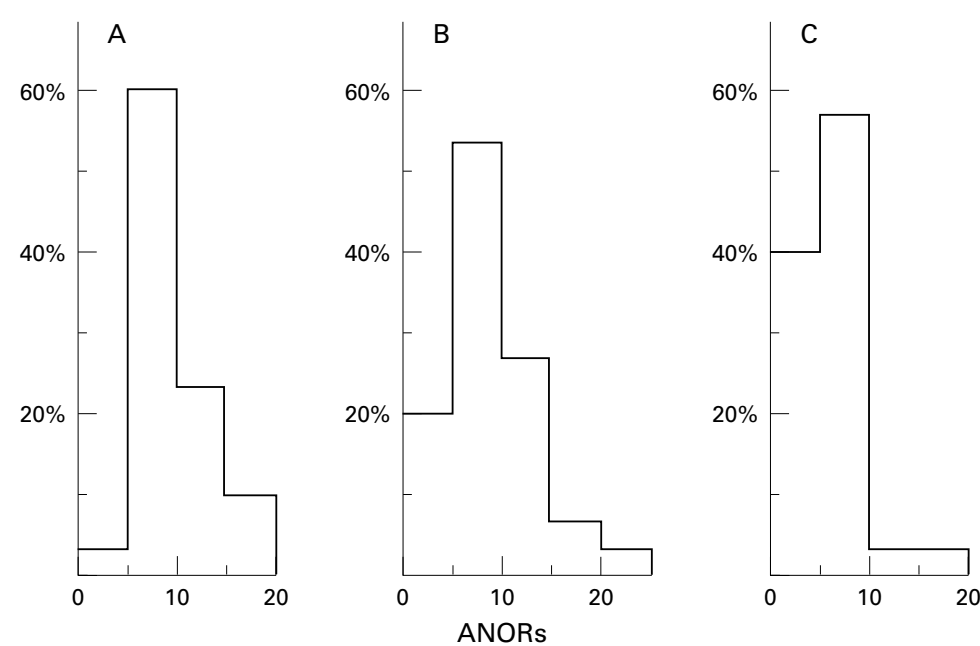

Figure 3 General distribution of cardiomyocytes with different AgNOR counts in nucleoli from ischaemic heart disease with NYHA II (A), III (B), or IV (C) functional stages of heart failure.
Table 1 Clinical details and the results of cardiomyocyte nucleolar silver staining

\begin{tabular}{llll}
\hline Group (n) & $\begin{array}{l}\text { NYHA } \\
\text { class }\end{array}$ & $\begin{array}{l}\text { Mean (SEM) } \\
\text { AgNORs }\end{array}$ & p value \\
\hline Control (8) & 0 & $8.0(0.4)$ & $<0.05$ \\
$\begin{array}{l}\text { Ischaemic heart disease } \\
\text { Stable course (10) }\end{array}$ & I/II & $9.9(0.7)$ & $<0.01$ \\
Stable course (5) & III & $8.8(0.5)$ & $<0.01$ \\
Unstable course (11) & III & $7.4(0.7)$ & $>0.05$ \\
Unstable course (5) & IV & $6.4(1.0)$ & \\
\hline
\end{tabular}

numbers of AgNORs in each nucleus were estimated. Comparison of mean values was performed by means of the Student's $t$ test.

\section{Results}

Some clinical details and the results of nucleolar silver staining of the myocardial samples from controls and from ischaemic heart disease cases are presented in table 1 and figs 2 and 3.

Both in normal and pathological cases, cardiomyocytes contained one, or occasionally, two or three silver stained nucleoli. The AgNOR scores in controls ranged from 7.5 to 12.5. The mean AgNOR score in cases with ischaemic heart disease not complicated by severe heart failure was higher than that in controls (mean (SEM), 9.9 (0.7) v 8.0 (0.4); $\mathrm{p}<0.05)$. There was also a difference in AgNOR numbers between cases with a stable or progressive form of ischaemic heart disease but the same NYHA functional stage (III) (mean (SEM) 8.8 (0.5) v 7.4 (0.7); p < 0.01). Analysis of cardiomyocyte distribution with different numbers of AgNORs showed that the latter associated negatively with the level of heart failure severity (fig 3 ). Thus, the activity of ribosomal cistrons in cardiomyocytes of patients with minimal and moderate signs of heart failure (NYHA class I/II; fig 3A) consisted mainly of moderately and highly active cells ( $84 \%$ and $12 \%$, respectively). In patients with NYHA class III heart failure (fig 3B), the histogram pattern is changed because of a decrease in the proportion of highly active cardiomyocytes and, in turn, an increase of those with relatively inactive nucleoli. In the case of severe heart failure (NYHA class IV) the proportion of relatively inactive cardiomyocytes is up to $40 \%$, while highly active cardiomyocytes disappeared completely (fig 3C).

\section{Discussion}

This study showed that the ribosomal RNA cistron activity in cardiomyocytes from ischaemic heart disease cases is relatively low. Compared with controls, it increased moderately only in cases with a benign course of the disease, not complicated by severe heart failure. However, the mean numbers of $\mathrm{Ag}$ NORs in cardiomyocyte nucleoli were significantly lower in cases with a progressive course of ischaemic heart disease, being the lowest in cases with severe heart failure. Because the heart samples were obtained within one hour of death, these findings are not related to 
postmortem artefacts. Furthermore, similar findings had been demonstrate previously by us using biopsy samples from patients with ischaemic heart disease. ${ }^{9}$

The mean number of AgNORs in cellular nuclei is known to be directly proportional to the concentration of RNA polymerase I, topoisomerase IIa, transcription factor $\mathrm{UBF}$, nucleolin, etc. ${ }^{11}$ Hence, it can be concluded that, in the course of ischaemic heart disease progression, ribosomal RNA cistron activity tends to decrease. This could be connected with a decrease in cardiomyocyte nuclear ploidy in ischaemic heart disease patients. ${ }^{12} \mathrm{On}$ the other hand, it might be explained by an adaptation of ischaemic heart disease cardiomyocytes to myocardium ischaemia at the level of pre-ribosomal RNA transcription.

Recently, in surgical patients, it has been shown that the content of basic contractile (myosin and actin) and cytoskeletal (desmin, titin, $\alpha$-activin, and vinculin) proteins in ischaemic heart disease cardiomyocytes is greatly diminished. ${ }^{6}$ Also, there is evidence that cardiomyocyte atrophy but not cardiomyocyte degeneration occurs in these cases. According to Schaper et al, depression of many (if not all) contractile and cytoskeletal proteins in ischaemic heart disease myocardium might result from a primary event at the transcriptional level. ${ }^{5}$ In relation to this hypothesis, there is evidence that concentrations of myosin, titin, and desmin mRNA are reduced in myocardium from patients with dilated cardiomyopathy, indicating that protein synthesis is disturbed at the transcription level. ${ }^{15}$ Our data show that the decrease in protein synthesis in ischaemic myocardium might be induced also at the ribosomal RNA cistron level. The significance of these molecular changes in ischaemic heart disease cardiomyocytes is not yet clear. Theoretically, it may represent a general adaptation of the myocardium to chronic ischaemia (a variant of hibernation after Ferrari $)^{13}$ at the level of ribosomal RNA cistron transcription and/or pre-ribosomal RNA processing. This might be more effective than at the level of translation. Evidently, such an indirect way of inhibiting general protein synthesis is sufficiently effective because it has not only been observed in human cardiomyocytes. A similar phenomenon of protein inhibition has been revealed recently in human collagen lattice cultured fibroblasts, where a decrease in fibrillarin gene expression and, hence, an impairment of pre-rRNA processing and ribosome formation took place. ${ }^{14}{ }^{15}$

Thus, we believe that the low concentration of proteins found in cardiomyocytes from cases with ischaemic heart disease may be a consequence of a general mechanism of cardiomyocyte adaptation to ischaemia because of ribosomal RNA synthesis inhibition. This mechanism appears to allow ischaemic myocardium to balance its work with the diminished level of circulation. However, the role of such a paradoxical adaptation of cardiomyocytes to ischaemia in ischaemic heart disease myocardium remains obscure. Indeed, ischaemia could contribute to the increase of cardiomyocyte protein deficiency, including contractile filaments and, hence, to further heart failure progression, myocardial ischaemia, and apoptosis. ${ }^{16}{ }^{17}$ As a result, the ribosomal RNA cistron activity of ischaemic heart disease cardiomyocytes should be progressively diminished, but this was not seen in our cases. Cytokines, such as tumour necrosis factor, which is produced excessively in the diseased myocardium, ${ }^{18}{ }^{19}$ might play an important role in cardiomyocyte metabolism under the influence of ischaemia.

In conclusion, the study of AgNORs shows the value of this method for the evaluation of myocardium under normal and pathological conditions. Also, it is shown that ischaemic myocardium from ischaemic heart disease cases might be an interesting subject for molecular biological investigations in relation to the basis of ischaemia.

1 Hein S, Fujitani N, Scholz D, et al. Transcription and translation of titin and alpha-actin are disturbed in diseased human myocardium. F Mol Cell Cardiol 1993;25(suppl 1):IIIP6.

2 Hein S, Scholz D, Fujitani N, et al. Altered expression of titin and contractile proteins in failing human myocardium. titin and Cell Cardiol 1994;26:1291-306.

3 Hein S, Scheffold Th, Schaper J. Ischaemia induces early changes to skeletal and contractile proteins in diseased human myocardium. F Thorac Cardiovasc Surg 1995;110: $89-8$

4 Hein S, Schaper J. Pathogenesis of dilated cardiomyopathy and heart failure: insights from cell morphology and biology. Curr Opin Cardiol 1996;11:293-301.

5 Schaper J, Hein S, Scholz D, et al. Multifaceted morphological alterations are present in the failing human heart. $\mathcal{F} \mathrm{Mol}$ Cell Cardiol 1995;27:857-61.

6 Schaper J, Hein S, Elsasser A, et al. Degeneration, apoptosis and necrosis - response to chronic heart disease of various causes [abstract]. F Mol Cell Cardiol 1996;28:A299.

7 Mamaev NN, Gudkova AY, Amineva KK, et al. A method for human cardiomyocyte protein-synthesising function assessment [Russian]. Arkh Anat Histol Embriol 1989;96: 69-72.

8 Gudkova AY, Amineva KhK, Mamaev NN. Nucleolar organizer activity of myocardial cells from patients with arterial hypertension of different genesis [Russian]. Arkh arterial hypertension

9 Mamaev NN, Kovalyeva OV, Amineva KhK, et al. Cardiomyocyte plastic function in surgical patients with ischaemic heart disease as evaluated by nucleolar silver staining [Russian]. Arkh Pathol 1992;54:43-5.

10 Howell WM, Black DA. Controlled silver staining of nucleolus organizer regions with a protective colloidal developer: a one step method. Experientia 1980;36:1014-5.

11 Roussel P, Hernandez-Verdun D. Identification of Ag-NOR proteins, markers of proliferation related to ribosomal gene activity. Exp Cell Res 1994;214:465-72.

12 Brodsky VYA, Sarkisov DS, Arefyeva AM, et al. Polyploidy in cardiac myocytes of normal and hypertrophic human

hearts; range of values. Virchows Archiv 1994;424:529-35. Ferrari R, Bongrazio M, Cargnoli A, et al. Heat shock protein changes in hybernation: a similarit

14 Gillary P, Georges N, Wegrowski J, et al. Protein synthesis in collagen lattice-cultured fibroblasts is controlled at the collagen lattice-cultured fibroblasts is cont
ribosomal level. FEBS Lett 1995;357:287-9.

15 Gillary P, Georges N, Randoux A, et al. Modulation of protein synthesis by extracellular matrix: potential involvement of two nucleolar proteins, nucleolin and fibrillarin. Biochem Biophys Res Commun 1996;228:94-9.

16 Narula J, Haider N, Virmani R, et al. Apoptosis in myocytes in end-stage heart failure. N Engl f Med 1996,335:1 182-9.

7 Olivetti G, Abbi R, Quaini F, et al. Apoptosis in the failing human heart. N Engl f Med 1997,336:1131-41.

18 Levine B, Kalman J, Mayer L, et al. Elevated circulating levels of tumor necrosis factor in severe chronic heart failure. N Engl F Med 1990;323:236-41.

19 Packer M. Is tumor necrosis factor an important neurohumoral mechanism in chronic heart failure? Circulation 1995;92:1379-82. 